\title{
In Reply to "Hydroxychloroquine Overdose: What Are the Exact Roles of Diazepam and Potassium Infusion?"
}

\author{
P. R. Chai ${ }^{1,2,3,4}$ - E. G. Ferro ${ }^{5}$ J. M. Kirshenbaum ${ }^{5}$ - B. D. Hayes ${ }^{6,7} \cdot$ S. E. Culbreth ${ }^{1,8} \cdot$ E. W. Boyer ${ }^{1,3} \cdot$ T. B. Erickson ${ }^{1,9}$
}

Received: 3 September 2020 / Revised: 3 September 2020 / Accepted: 4 September 2020 / Published online: 14 September 2020

(C) American College of Medical Toxicology 2020

We thank Drs. Megarbane and Schicchi for their thoughtful comments and highlights regarding recent animal models suggesting the importance of mechanical ventilation and vasopressor support in the setting of acute hydroxychloroquine poisoning [1, 2]. We agree that although the literature on hydroxychloroquine poisoning is scarce, it is reasonable to infer treatment strategies from clinical experience with chloroquine ingestions.

Drs. Megarbane and Schicchi make valid points on the consideration of initiation of intravenous diazepam compared with epinephrine infusions in moderately and severely poisoned patients. The writers point to in vitro and in vivo data suggesting that the use of diazepam does not impact cardiac performance nor do its attributable cardioprotective effects contribute to significant recovery of cardiac function in poisoned rats $[1,3]$. These investigations do suggest that diazepam may have some effect in augmenting the inotropy imparted through concomitant administration of vasopressors. It does, however, highlight the challenge in developing data-driven treatment algorithms for acutely poisoned patients given the lack of ethical mechanisms through which to conduct adequately powered human clinical trials. Clinically, one confounding factor frequently encountered is the unknown dose of hydroxychloroquine taken and the time of ingestion, as well as coingestants. While hydroxychloroquine (HCQ) levels might suggest the severity of ingestion, if we

Supervising Editor: Lewis Nelson, MD

P. R. Chai

pchai@bwh.harvard.edu

1 Division of Medical Toxicology, Department of Emergency Medicine, Brigham and Women's Hospital, Boston, MA 02115, USA

2 Department of Psychosocial Oncology and Palliative Care, Dana Farber Cancer Institute, Boston, MA, USA

3 The Fenway Institute, Boston, MA, USA

4 The Koch Institute for Integrated Cancer Research, Massachusetts Institute of Technology, Boston, MA, USA extrapolate from data surrounding chloroquine, these concentrations do not result in real time and are rarely clinically relevant once obtained. We therefore think that in some cases, diazepam may still be of value, especially in critically ill individuals who are mechanically ventilated, supported hemodynamically with vasopressors, and continue to manifest signs of HCQ toxicity. Unlike animal studies that have defined doses and controlled times to intervention, the condition of human intentional and unintentional ingestion is widely variable, often associated with chronic toxicity, coingestants, and underlying disease states, thereby requiring elasticity around direct extrapolation of animal data. In individuals who have moderate HQC toxicity (acute doses of 2-4 g), diazepam may be less effective, assuming that the dose ingested is known [2].

We also thank Drs. Megarbane and Schicchi for reminding us that correcting hydroxychloroquine-induced hypokalemia should be a careful intervention as aggressive over-correction can result in hyperkalemia. Depending of the degree of cardiac conduction abnormalities seen after acute hydroxychloroquine overdose, infusion of potassium may help eliminate the confounding factor of hypokalemia contributing to dysrhythmias. As medical and clinical toxicologists, we frequently manage the effects of druginduced hypokalemia due to intracellular sequestration of potassium ions. Ensuring correction of this electrolyte abnormality and

5 Department of Medicine, Brigham and Women's Hospital, Boston, MA, USA

6 Department of Emergency Medicine, Harvard Medical School, Boston, MA, USA

7 Department of Pharmacy, Massachusetts General Hospital, Boston, MA, USA

8 Department of Pharmacy, Brigham and Women's Hospital, Boston, MA, USA

9 Harvard Humanitarian Institute, Boston, MA, USA 
understanding the potential for rebound hyperkalemia is critical in the safe management of hydroxychloroquine poisoning.

Overall, in a critically ill individual suffering from acute hydroxychloroquine overdose, the use of vasopressors, mechanical ventilation, and possibly diazepam are features of management that should be considered during the clinical course [3]. The management of hydroxychloroquine-induced dysrhythmias may need to include correction of hypokalemia and in the setting of a prolonged QRS complex and consideration of sodium loading agents like hypertonic saline and sodium bicarbonate. There is clearly a need for additional investigations, both in animals and potentially on human subjects to understand the impact of these various treatment options to better address this potentially lifethreatening poisoning that has become increasingly relevant during the COVID-19 pandemic [4].

Funding None.

\section{Compliance with Ethical Standards}

Conflict of Interest PRC was funded by NIH K23DA044874, R44DA051106 investigator-initiated research grants from E Ink
Corporation, Hans and Mavis Psychosocial Foundation, Philips Biosensing, and Gilead Sciences; EWB was funded by R01DA047236.

\section{References}

1. Hughes DA. Acute chloroquine poisoning: a comprehensive experimental toxicology assessment of the role of diazepam. $\mathrm{Br} J$ Pharmacol. 2020. https://doi.org/10.1111/bph.15101.

2. Clemessy JL, Angel G, Borron SW, et al. Therapeutic trial of diazepam versus placebo in acute chloroquine intoxications of moderate gravity. Intensive Care Med. 1996;22:1400-5. https://doi.org/10. 1007/bf01709558.

3. Riou B, Lecarpentier Y, Barriot P, et al. Diazepam does not improve the mechanical performance of rat cardiac papillary muscle exposed to chloroquine in vitro. Intensive Care Med. 1989;15:390-5. https:// doi.org/10.1007/bf00261499.

4. Edwards E and Hillyard V. Man dies after taking chloroquine in an attempt to prevent coronavirus, https://www.nbcnews.com/health/ health-news/man-dies-after-ingesting-chloroquine-attempt-preventcoronavirus-n1167166 (2020, accessed March 27, 2020 2020).

Publisher's Note Springer Nature remains neutral with regard to jurisdictional claims in published maps and institutional affiliations. 